\title{
Structural Join Algorithm for Sequential Regular Path Expressions
}

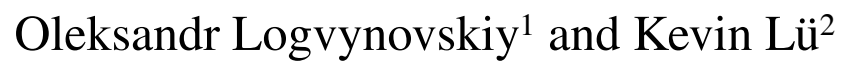 \\ ${ }^{1}$ SCISM, South Bank University, London, UK \\ ${ }^{2}$ School of Business and Management, Brunel University, Uxbridge, UK
}

\begin{abstract}
XML queries employ regular path expressions to find structural patterns within XML documents. The operation of structural join is a crucial part of XML query processing. Existing approaches reduce complex join expressions to several binary structural joins. It implies generation of superfluous intermediate data. In this paper, we propose a new structural join algorithm, called sequence join algorithm, for sequential regular path expressions. It exploits information about position of the elements in the document to skip generation of the redundant intermediate lists. The algorithm performs merge of several input lists of nodes in one pass. Experimental results prove the algorithm is better than multiple binary join algorithm for queries of both small and large cardinality.
\end{abstract}

Keywords: XML document, regular path expression, structural join algorithm.

\section{Introduction}

Extensible Markup Language (XML) is now used as the de facto means to handle semistructured data over the Web [1]. Semistructured data arises when a data source does not impose a rigid structure and/or data is combined from several heterogeneous sources. The power of XML is in its ability to describe hierarchical structures and extend the dictionary of available data types.

XML documents are described in terms of elements [8]. The elements are enfolded by tags, which represent their type names. Each element is either treated as a container for some other elements or associated with an atomic value (such as text, multimedia content, etc.). Structural relationships among the elements are defined by nesting (containment) of the elements or by referencing.
A query over the XML data specifies structural patterns among the elements in the document. The result of such a query is intended to locate all occurrences of these patterns within the XML document or database. Such patterns are also known as regular path expressions (RPEs) and constitute the basis for the statements of XML query languages, for instance XQuery [11], Lorel [5], XML-QL [7], XPath [9], etc. For example, the XQuery path expression $/ /$ project/task/resource/name specifies the retrieval of the names of all resources assigned to the tasks of the project.

The specified structural patterns are often complex themselves, but can be decomposed into a set of basic structural relationships between elements. Finding matches of the query against the database can then be considered as matching each of the basic structural relationships against the data and following merging of the sub-results. This process is performed by means of a structural join operation [2]. The operation essentially depends on finding ancestor-descendant and parent-child relationships among nodes of the semistructured database. The effective implementation of the structural join operation over ancestor-descendant (parentchild) pattern is the crucial part of XML query processing.

The recently proposed join algorithms take advantage of the element numbering to compute ancestor-descendant (parent-child) relationship. The element numbering allows uniquely identify the element position within the XML database. The position of the element can be described by 4-tuple (DocID, StartPos, EndPos, Level). DocID is the identifier of the XML 
document within the XML database; StartPos, EndPos are the text positions of the first and the last character of the element within the document respectively, and Level is the nesting depth of the element within the document [6]. The alternative representations of the element position preserve the DocID and Level components of the tuple, but differ in the way to define the start and end positions within the document. For example, there is another representation (DocID, PrePos, PostPos, Level), which uses the position numbers assigned to the element by pre-order (PrePos) and postorder (PostPos) traversals of the document tree accordingly. The other approaches are equivalent to the latter one and can be transformed by appropriate mapping.

The key idea underlying the implementation of the existing join algorithms is the decomposition of the original query path expression into a set of simple (binary) path expressions. Each binary expression produces an intermediate join result, which is used on the subsequent stage. For example, the path expression //project/task/resource/name can be decomposed to project/task and resource/ name. Then the intermediate results are joined together. At each of the stages, the join algorithm uses element numbering to check the ancestor-descendant or parent-child relation between the nodes.

The XISS system [3] introduces three join algorithms: element-attribute (EA-join), elementelement (EE-join), and Kleene-closure (KCjoin). The element-attribute algorithm joins two intermediate results from subexpressions, which are a list of elements and a list of attributes. The element-element algorithm joins two lists of elements. The principal difference between these algorithms is that the latter one checks ancestor-descendant relationship between each pair of the input lists while the former one tests parent-child relationship. The Kleene-closure algorithms iteratively use element-element algorithm to compute closure of the expression. It repeatedly applies EE-join to the result from the previous stage of iteration. Both EA-join and EE-join algorithms have a loop over one input list nested into a loop over another list and, therefore, have time complexity $O\left(\left|E_{1}\right| \cdot\left|E_{2}\right|\right)$, which is quadratic in the size of the input lists. As $\mathrm{KC}$-join depends upon EE-join, it also has quadratic time complexity.
Structural join algorithms proposed by Al-Khalifa et al. [2] exploit the advantage of element numbering to decrease the time of processing. The tree-merge join algorithm is an extension of relational equality merge join performed on sorted inputs. It was adopted to deal with ancestor-descendant or parent-child tests. The time complexity of the tree-merge join is non-quadratic $O\left(\left|E_{1}\right|+\left|E_{2}\right|\right)$, but may include multiple passes over the same input set of descendant nodes. To avoid this problem, the second of the proposed algorithms, stack-tree join algorithm, utilises stack of nodes and has time complexity $\left.O\left(\left|E_{1}\right|+\left|E_{2}\right|\right) / B\right)$, where $B$ is the blocking factor.

The main drawback of the considered algorithms is their limitation to merge only two input lists per join. It means that for a sequential regular path expression there may be generated several intermediate results. For example, processing the path expression //project/task/ resource/name results in two joins (project/ task) and (resource/name). Then, yet another join is performed to merge intermediate results. This requires additional time to create and scan intermediate data: two intermediate tables will be created and scanned. This factor becomes even more important when processing big semistructured databases. Our approach allows to reduce intermediate processing by simultaneous merging of several inputs. For the given example, it will require only one join operation instead of three.

Bruno et al. have extended the structural join algorithm to avoid multiple binary joins and further merge of intermediate results [10]. The introduced twig join algorithm uses stacks to store elements of the paths successfully tested against a query. Although this is similar to our approach, we exploit numbering, not only for elimination of unnecessary ancestor-descendant checks within the current element subtree, but for fast skipping of entire subtrees.

The main goal of the paper is to introduce an effective structural join algorithm for sequential regular path expressions, which takes several sets of single-element subexpressions as an input. It exploits the element numbering and performs merging of all the input sets in one pass. We present experimental results performed by our prototype system over several real and synthetic datasets. They show that the proposed algorithm can process regular path expressions faster than other algorithms. 
The rest of the paper is organised as follows. Section 2 presents background material (data model, query expressions, structural relationships and element numbering). In section 3 we develop the structural join algorithm for regular path expressions. Section 4 addresses experimental aspect and evaluates the proposed algorithm. Section 5 summarises results of the paper and discusses future work.

\section{Background and Overview}

The XML data can be modelled either as a tree or as a graph. The tree model reflects the logical structure of an XML document. Nodes of the tree correspond to the elements of the document. Nesting of the elements is reflected by the parent-child relationships among nodes. Some of the tree nodes have as their values references to other elements (usually in form of IDREF attributes). The graph model extends the tree by treating such reference nodes as arcs [5]. Although the graph model is more powerful than the tree, it challenges more problems, like cyclereferences.

As this paper concentrates on handling ancestordescendant relationship, we use a tree data model. The tree model can be used both to store and query semistructured data [2], [3]. Within the XML database, the order numbers of the tree node, along with its depth level and document number, are used to find the structural relationships between these nodes. This section describes the tree data model and introduces basic structural relationships used for querying XML documents. Then it explains the node position notion and its usage for finding structural relationships between the nodes.

\subsection{Data Model}

An XML database is a forest of trees corresponding to each document within the database. An XML document is a rooted, ordered, labelled tree. Each element of the document forms a node of the tree labelled with the element type (tag name) and value. The edges of the tree stand for parent-child (containment) relationship between the elements. All subelements nested within the element appear in the tree as the child nodes directly connected with the edges to a parent node. Attributes of the element are represented similarly to nested subelements and form additional nodes in the tree, emanating from their associated parent nodes.



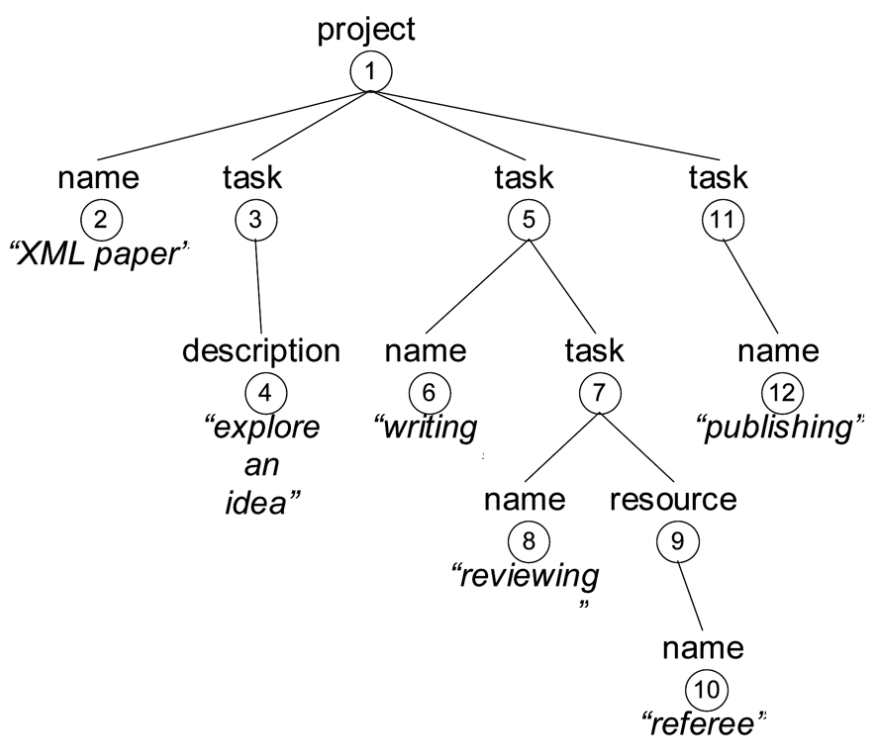

b)

Fig. 1. Example XML data (a) and its corresponding tree (b). 
An example of the XML data is shown in Figure 1(a) and its tree representation is shown in Figure 1(b).

The XML data tree has an implicit order of its nodes. The total order of all the nodes in the tree is obtained by a pre-order tree traversal (a depth-first, left-to-right traversal of the nodes). The nodes order of our example tree is shown in Figure 1(b) within the circles of corresponding nodes.

We exploit the order of the nodes in the data tree to compute position of the element in the database. A position of the element is a 4-tuple (DocID, StartInt, EndInt, Level). DocID is the identifier of the XML document within the XML database, StartInt is the tree order number of the node corresponding to the element, EndPos is the tree order number of the last descendant of the node, and Level is the nesting depth of the node within the tree. For the given example in Figure 2, the positions of the nodes are shown within rectangles. Consider, for instance, the node "resource". Its first position number stands for document identifier and equals to 1 . The second and third position numbers represent the interval of the node $[9,10]$ : order number of the node itself $(9)$ and order number of the last descendant (10), node "name/"referee"". The last position number shows depth of the node within the tree and equals to 4 . The position numbers of all other nodes are computed in the analogous way.

\begin{tabular}{ll}
\hline \hline axis name & description \\
\hline ancestor & ancestors of the node \\
attribute & attributes of the node \\
child & children of the node \\
descendant & descendants of the node \\
following & all nodes after the node \\
parent & parent of the node \\
preceding & all nodes before the node \\
self & node itself \\
\hline \hline
\end{tabular}

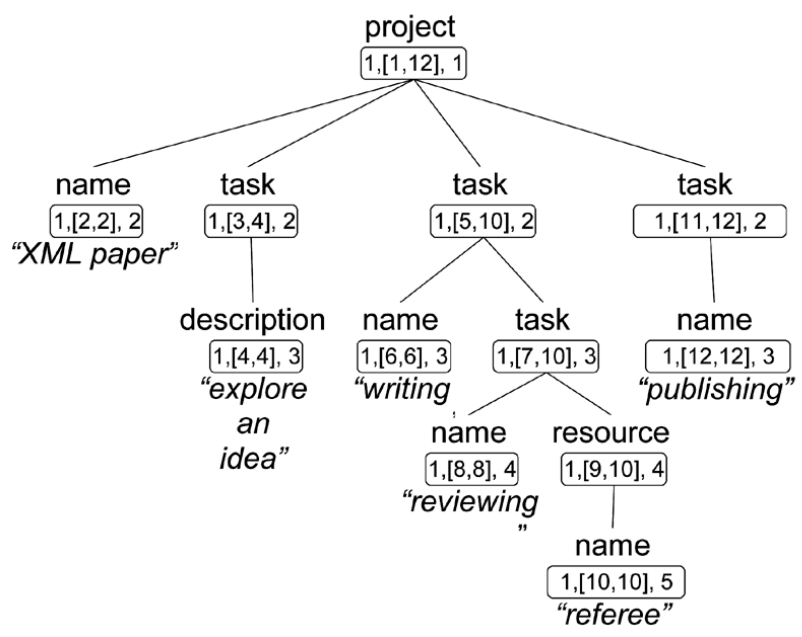

Fig. 2. Positions of the elements.

The position of the node is used to determine structural relationships between any two nodes of the tree.

\subsection{Structural Relationships}

Relationships between a node and the other nodes in the tree are not limited by implicit containment (parent-child) relationship and described by axes of the node [11]. An axis is a relation that parts the set of all the tree nodes into subsets with respect to the current node. The most commonly used axes of the node are summarised in Figure 3 (a).

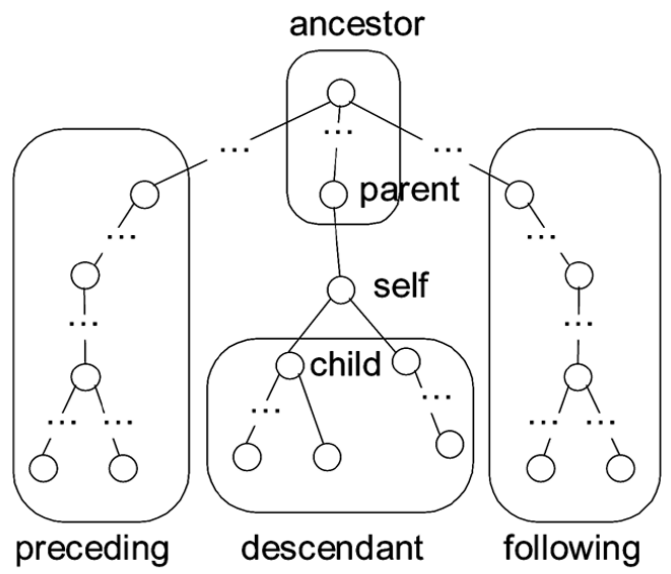


The ancestor, descendant, following, preceding, and self axes partition document nodes into nonoverlapped subsets, as it is shown in Figure 3 (b). The child axis is the subset of the descendant axis and the parent axis is the subset of the ancestor axis.

A regular path expression or location path of a target node B from source node A (hereafter referred to as path) is a sequence of nodes in the tree, from the node A to the node B. Relationships between intermediate nodes in the path are described by axes. A location path is called absolute path if its source node is the root of the tree, otherwise it is called relative path.

Regular path expressions are an essential component of semistructured data query languages (e.g. XQuery [11]), which utilise variety of structural relationships between the nodes.

\subsection{Handling Structural Relationships by Means of Position}

Position is an important characteristic of elements within XML documents and it is intensely used for indexing and querying semistructured data.

The position of the node $n_{i}$ is denoted as $\left(D_{i},\left[S_{i}\right.\right.$, $\left.\left.E_{i}\right], L_{i}\right)$, where $D_{i}$ is the identifier of the XML document within the XML database; $S_{i}$ is the tree order number of the node $n_{i}, E_{i}$ is the tree order number of the last descendant of the node $n_{i}$, and $L_{i}$ is the nesting depth of the node $n_{i}$ within the tree. The pair of the node order $S_{i}$ and order of its last descendant $E_{i}$ constitutes an interval of descendant order numbers $\left[S_{i}, E_{i}\right]$. Hereafter, we will refer to the interval $\left[S_{i}, E_{i}\right]$ as the node interval.

Ancestor-descendant relationship. Given a tree node $n_{i}$ and its position $\left(D_{i},\left[S_{i}, E_{i}\right], L_{i}\right)$ and a tree node $n_{j}$ and its position $\left(D_{j},\left[S_{j}, E_{j}\right], L_{j}\right)$, the node $n_{i}$ is an ancestor of the node $n_{j}$ (and node $n_{j}$ is a descendant of the node $n_{i}$ ) iff:

a) $D_{i}=D_{j}$, i.e. both nodes belong to the same document;

b) $\left[S_{i}, E_{i}\right] \subset\left[S_{j}, E_{j}\right]$, the node interval of the ancestor includes the interval of the descendant, i.e. $S_{i}<S_{j}$ and $E_{i}>E_{j}$.

Parent-child relationship. Given a tree node $n_{i}$ and its position $\left(D_{i},\left[S_{i}, E_{i}\right], L_{i}\right)$ and a tree node $n_{j}$ and its position $\left(D_{j},\left[S_{j}, E_{j}\right], L_{j}\right)$, the node $n_{i}$ is a parent of the node $n_{j}$ (and node $n_{j}$ is a child of the node $n_{i}$ ) iff:

a) $D_{i}=D_{j}$, i.e. both nodes belong to the same document;

b) $\left[S_{i}, E_{i}\right] \subset\left[S_{j}, E_{j}\right]$, the node interval of the ancestor includes the interval of the descendant, i.e. $S_{i}<S_{j}$ and $E_{i}>E_{j}$;

c) $L_{i}=L_{j}-1$, the descendant is nested immediately within the ancestor node.

For instance, consider the nodes "project" and "resource" in Figure 2. Their document ID numbers coincide and equal to 1 . The node interval of "project" includes the interval of the "resource", $[1,12] \subset[9,10]$, so the "project" is the ancestor of the "resource". The difference between their levels exceeds 1 , so they are not bounded by parent-child relationship.

\begin{tabular}{llll}
\hline \hline relation & Document & Node interval & Level \\
\hline ancestor & $D_{i}=D_{j}$ & {$\left[S_{i}, E_{i}\right] \subset\left[S_{j}, E_{j}\right]$} & $L_{i}<L_{j}$ \\
attribute & $D_{i}=D_{j}$ & {$\left[S_{i}, E_{i}\right] \supset\left[S_{j}, E_{j}\right]$} & $L_{i}=L_{j}+1$ \\
child & $D_{i}=D_{j}$ & {$\left[S_{i}, E_{i}\right] \supset\left[S_{j}, E_{j}\right]$} & $L_{i}=L_{j}+1$ \\
descendant & $D_{i}=D_{j}$ & {$\left[S_{i}, E_{i}\right] \supset\left[S_{j}, E_{j}\right]$} & $L_{i}>L_{j}$ \\
following & $D_{i}=D_{j}$ & {$\left[S_{i}, E_{i}\right]>\left[S_{j}, E_{j}\right]$} & \\
parent & $D_{i}=D_{j}$ & {$\left[S_{i}, E_{i}\right] \subset\left[S_{j}, E_{j}\right]$} & $L_{i}=L_{j}-1$ \\
preceding & $D_{i}=D_{j}$ & {$\left[S_{i}, E_{i}\right]<\left[S_{j}, E_{j}\right]$} & \\
self & $D_{i}=D_{j}$ & {$\left[S_{i}, E_{i}\right]=\left[S_{j}, E_{j}\right]$} & $L_{i}=L_{j}$ \\
\hline \hline
\end{tabular}

Table 1. Summary of position constraints to represent structural relationships. 
The other structural relationships between any two nodes can be verified by analogy. They are summarised in Table 1.

In Figure 2, the node "description" precedes the node "resource" as they have the same document number and the interval of the "description" occurs before the interval of the "resource", $[4,4]<[9,10]$.

The operational cost to check any of the structural relationship includes document ID, node interval, and level comparison. The costs are equal for any type of relationship, but the ancestor-descendant and parent-child are the ones mostly used.

\section{Algorithm}

In this section, we develop new structural join algorithm, in order to efficiently process regular path expression queries. It exploits the concept of node position to merge several input lists in one pass. As it processes several binary structural relationships that form a sequence, we call it sequence join algorithm.

At the outset, we consider two ways of result representation (a tree-like form and tuples) and show the algorithm in action on a detailed example. Then we present algorithm itself and conclude with analytical estimation of its performance.

\subsection{Representation of the Results}

The results of the XML query can be represented either as a tree or as a list of tuples. Consider the following query example //project// task//name performed over the data shown in Figure 2. The query retrieves the names of the elements assigned to all tasks within the project (including names of the tasks themselves). The tree and the tuples of the result are shown in Figure $4 \mathrm{a}$ ) and b) respectively.

These two forms are equivalent and can be transformed from one to another. The tree representation can be obtained from the tuples by eliminating duplicates of the nodes.

The tree view is more compact and suitable for the data that originally has tree-like structure. Nevertheless, it may fit in applications that need

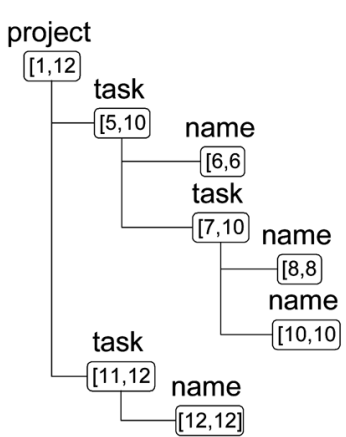

a)

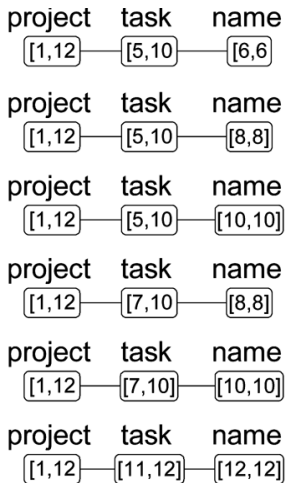

b)
Fig. 4. Forms of query results: tree and tuple.

results in a table-like form. In such a case, tuple representation is more appropriate. Final decision on which of the two forms is preferable should be made for each particular case.

Hereafter, we use tree representation of the results, adding, if necessary, some comments on the tuple form.

\subsection{Example}

For the purpose of clarity, we demonstrate the algorithm on an example, before introducing it more formally.

Consider the query expression //project// task//name applied to the data in Figure 2. The simplified data tree is presented in Figure 5 a). The document number and level components of the node position are omitted. The document number is equal for all the nodes of the example and level is not important as no parent-child relationship is tested through the query processing. The input lists of the example are shown in Figure $5 \mathrm{~b}$ ). The list $A_{1}$ represents the result of select operation and contains all nodes "project" selected from the database. Analogously, the lists $A_{2}$ and $A_{3}$ include nodes "task" and "name" respectively.

The steps of the algorithm for the chosen example are shown in Figure 6. The current node of each list is marked by arrow. For each input list, the algorithm keeps value of a current node range of the list. The range points to candidate nodes to be added to the result list. It coincides with the node interval component of the node position, but covers only the nodes from the 


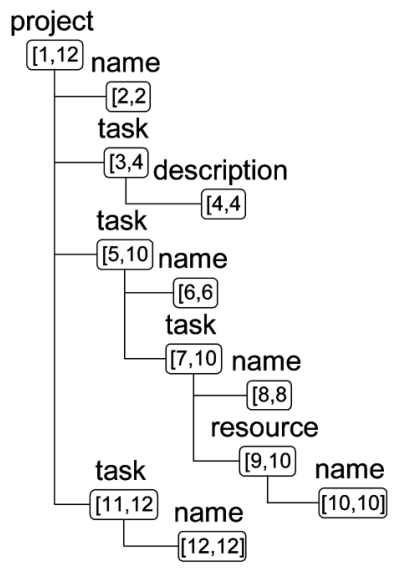

a)

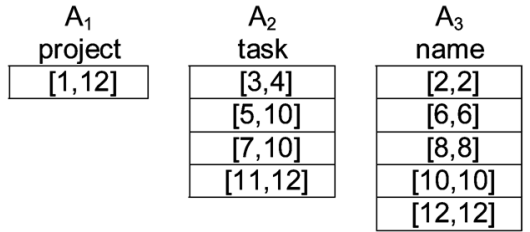

b)

Fig. 5. Example of data tree and input lists.

same list. For example, the interval of the node "task $[5,10]$ " in Figure 5 a) is equal to $[5,10]$ and includes six nodes 5 to 10 . The range of the same node is equal to $[5,10]$ too, but includes only two "task" nodes 5 and 7. In Figure 6, the range is shown in grey colour. Additionally, each of the lists is visually extended with empty cells to show precedence of the nodes in the source tree. For example, the range $[2,2]$ of the list $A_{3}$ in Figure 6 a) is preceding the range
[3,4] of the list $A_{2}$ and is part of (included into) the range $[1,12]$ of the list $A_{1}$.

The basic idea of the algorithm is to synchronously read input lists to find first match of the ranges. Once the matching ranges are found, the current nodes within ranges are put into the result list. If the ranges in two adjacent lists do not match, then one of the ranges changes, based on the result of their comparison. The propagation of changes goes from the last list

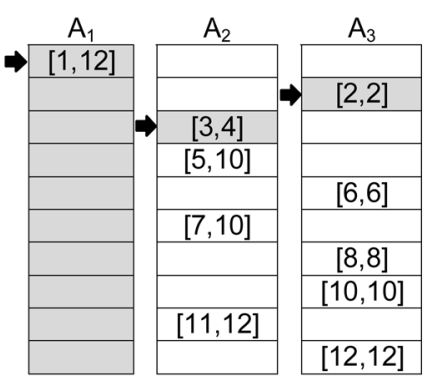

a)

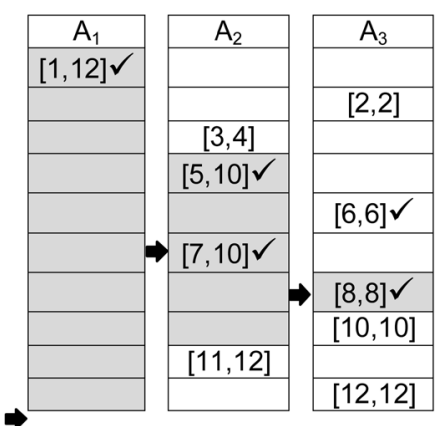

d)

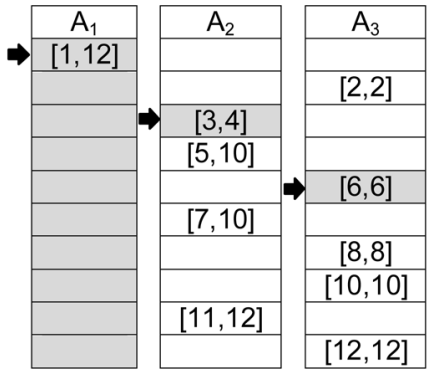

b)



e)

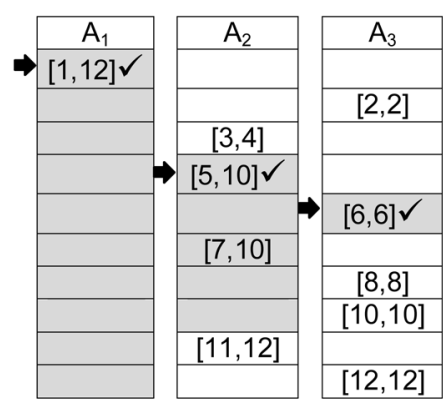

c)

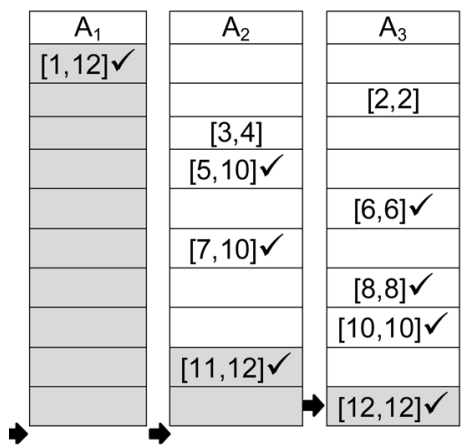

f)

Fig. 6. Step-by-step example. 
back to first. The algorithm stops when no more matches of the ranges can be found.

For the given example, the algorithm starts from the state in Figure 6 a). The ranges are initially assigned to the first nodes of the lists. The comparison of the ranges goes from the list of ancestors to the list of descendants (left-to-right in the figure). The range $[3,4]$ of the descendant list $A_{2}$ satisfies the range $[1,12]$ of the ancestor list $A_{1}$, but the range [2,2] of the descendant list $A_{3}$ is not included into the range [3,4] of the ancestor list $A_{2}$. As the descendant range [2,2] precedes the ancestor range $[3,4]$, the algorithm reads a new node from the list $A_{3}$ and changes range to $[6,6]$, as it is shown in Figure $6 \mathrm{~b}$ ).

Now the descendant range $[6,6]$ follows the ancestor range $[3,4]$. Therefore, the latter is changed to $[5,10]$ (Figure $6 \mathrm{c}$ ). At this point, all descendant ranges satisfy the ancestor ranges and their nodes (up to current) are sent to the output list. The nodes added to the output are marked with the tick mark. The algorithm reads new nodes from the lists and changes ranges (Figure $6 \mathrm{~d}$ ). For the list $A_{3}$, the range is changed from $[6,6]$ to $[8,8]$. The range of the list $A_{2}$ is not changed as the new node $[7,10]$ is still within the current range. The range of the list $A_{1}$ is not changed in spite the fact that it has reached the end of the list. This is due to the assumption that propagation of changes goes from the last list to the first. The nodes $[8,8]$ and $[7,10]$ are sent to the output.

By analogy to the previous step, the algorithm continues reading the lists (Figure $6 \mathrm{e}$ ). For the list $A_{3}$, the range is changed from $[8,8]$ to $[10,10]$. The range $[5,10]$ of the list $A_{2}$ is not changed as the range of the list $A_{3}$ still satisfies it and no propagation is necessary. The node $[10,10]$ is added to the result.

The next read operation of the node $[12,12]$ of the list $A_{3}$ forces to change the range of the list $A_{2}$ from $[5,10]$ to $[11,12]$ (Figure $6 \mathrm{f}$ ). Nodes $[11,12]$ and $[12,12]$ are appended to the output list. Any further reading from the list $A_{3}$ fails and the algorithm stops.

\subsection{Algorithm of Sequence Join}

The text of the algorithm is presented in Figure 7. The steps of finding match of the ranges and appending result nodes are presented as separate sub-routines.

The algorithm generates the result list in the tree representation. To generate tuple representation the append subroutine should iterate over the range nodes producing each possible combination.

The algorithm uses recursive subroutines. The depth of the recursion is equal the number of input lists which, in its turn, is related the depth of the input XML data tree. The depth of the existing XML databases does not exceed 20 and recursion will not cause consumption of memory resources.

\subsection{Time Cost Estimation}

In this section we estimate the execution time difference between the proposed join algorithm and the pair-wise join algorithm. As there are multiple ways to break and n-way join into $n$ binary joins, and the decision is chosen at run time, we assume that it is performed sequentially and all intermediate results are materialised.

Consider regular path expression $a_{1} / a_{2} / \ldots / a_{n}$. Both approaches require $\mathrm{n}$ selection operators resulting in lists of nodes $A_{1}, A_{2}, \ldots, A_{n}$ respectively. The execution plans for pair-wise join and sequence join are shown in Figure 8 a) and $b$ ) accordingly. We consider the total time to perform join operation as the sum of time needed to read input lists $(\sigma)$ and time necessary to create output list $(\tau): \sigma+\tau$.

For pair-wise approach, the task of matching complex query is reduced to performing of one join operation for each binary structural relationship in query expression. For $n$ input lists of nodes, it causes creation of $\frac{n}{2}$ intermediate lists of nodes. The next step is to perform binary join operation over the intermediate lists. It is applied until it results in the only one, result list. Thus, the whole number of intermediate list (including the result list) is $\frac{n}{2}+\frac{n}{4}+\ldots+\frac{n}{2^{\log n}}=$ $\left(1-\frac{1}{2^{\log n}}\right) n=n-1$. In Figure 8 a) these lists are denoted as $A_{n+1}, \ldots, A_{2 n-1}$. If $\tau_{i}$ is the time required to create list $i$, then the total time necessary for creation of intermediate lists is $\sum_{n+1}^{2 n-1} \tau_{i}$ 


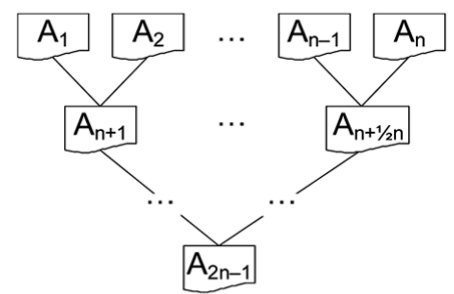

a)

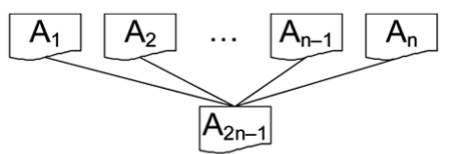

b)

Fig. 8. Execution plans of multiple pair-wise join and sequence join.

and read list $i$ respectively.

The sequence join reads input lists $A_{1}, \ldots, A_{n}$ and creates the only one, result list $A_{2 n-1}$, as shown in Figure $8 \mathrm{~b}$ ). The total time necessary to perform sequence join is

$$
\sum_{1}^{n} \sigma_{i}+\tau_{2 n-1}
$$

where $\sigma_{i}$ is the time required to read input lists, and $\tau_{2 n-1}$ is the time needed to create result table.

The time difference between the two approaches is

$$
\begin{gathered}
\left(\sum_{1}^{2 n-2} \sigma_{i}+\sum_{n+1}^{2 n-1} \tau_{i}\right)-\left(\sum_{1}^{n} \sigma_{i}+\tau_{2 n-1}\right) \\
=\sum_{n+1}^{2 n-2} \sigma_{i}+\sum_{n+1}^{2 n-2} \tau_{i},
\end{gathered}
$$

where $\tau_{i}$ and $\sigma_{i}$ is the time required to create and read list $i$ respectively.

The parameters $\tau_{i}$ and $\sigma_{i}$ depend on the capacity of the list $i$. If the number of nodes in each list is comparable, then we can assume the times to create and read list are equal: $\forall i, i=1, \ldots, n$; $\tau_{i}=\tau$. The time cost functions of the algorithms (1) and (2), as well as their difference (3), can be represented in a simpler form:

the time cost of multiple pair-wise join is

$$
(2 \sigma+\tau)(n-1)
$$

the time cost of sequence join is

$$
(\sigma n+\tau)
$$

and their difference is

$$
(\sigma+\tau)(n-2)
$$

where $\tau$ and $\sigma$ is an average time required to create an output and read an input list respectively, $n$ is the number of original input lists.

The algorithm time cost graphs are presented in Figure 9.

The graph shows that the sequence join algorithm does better over the queries with 3 and more basic structural relationships.

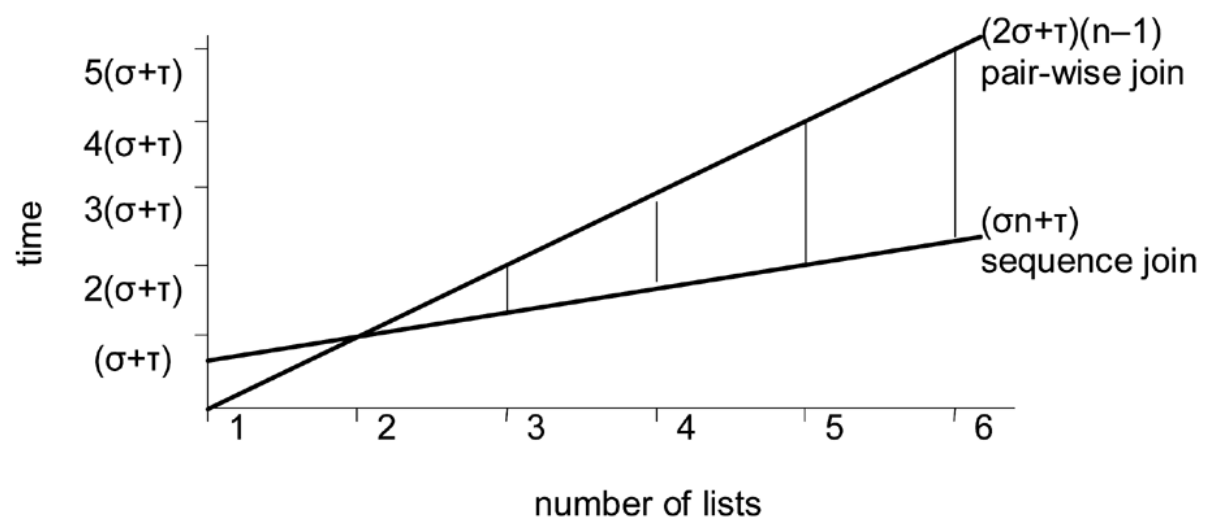

Fig. 9. Time cost difference between the pair-wise join and sequence join. 


\section{Experimental Evaluation}

We have conducted several experiments evaluating the proposed algorithm. The tests involve both real-world and synthetic datasets. This section describes experimental testbed and presents the results of these experiments.

\subsection{Experimental Setup}

We implemented a prototype system for storing, indexing, and querying XML data. The screenshot of the prototype window is shown in Figure 10. Source XML files are parsed using the Xerces-C ++ XML parser [17]. The data is parsed without validation option. The prototype exploits Berkeley DB [18] to store parsed data and index files. The B-tree indexing facility of the package is used to build index files. The system provides a simple query interface for regular path expressions. The interface is XQuery-compliant [11] and directly performs query processing. The overall system is implemented in $\mathrm{C}++$.

All experiments were performed under Windows 2000 workstation software running on a
Dell Dimension 8100 computer. The station has $256 \mathrm{Mb}$ of memory, $20 \mathrm{~Gb}$ hard disk, and 1.4 $\mathrm{GHz}$ Pentium IV processor.

\subsection{Data Sets}

We have chosen several data sets, both realworld (DBLP [12], Shakespeare [13], HAMRADIO [14], Mondial [15]), and synthetic (Xmark [16]). The characteristics of these sources of data are summarised in Table 2. The columns Files and Size of the Source data section show the number of the source XML documents within the dataset and disk space occupied by them respectively. The field Size of the Database section refers to the size of the database obtained after parsing and storing the source documents. The Element column represents the number of different element type names. It includes all the attribute names. The Occurrences field indicates the total number of element occurrences within the document and includes all attribute occurrences as well. The column values show the total number of different values of the elements. The field Depth show the maximum level of element nesting.

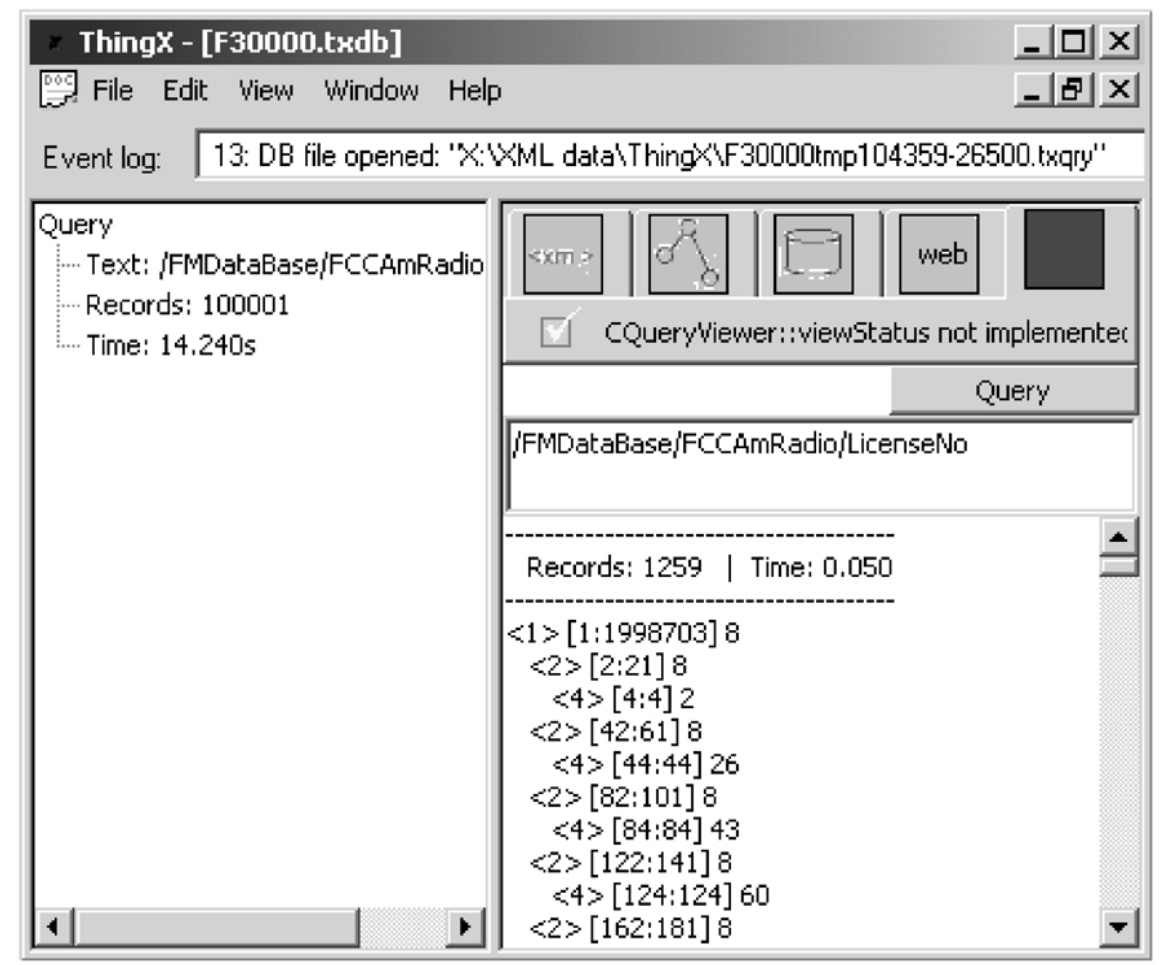

Fig. 10. Prototype system window. 


\begin{tabular}{l|r||r|r||r|r|r|r|r}
\hline \multirow{2}{*}{$\begin{array}{c}\text { XML } \\
\text { documents }\end{array}$} & \multirow{2}{*}{ Type } & \multicolumn{2}{c||}{ Source data } & \multicolumn{5}{c}{ Database data } \\
\cline { 3 - 9 } & & Files & Size $(\mathrm{Mb})$ & Size $(\mathrm{Mb})$ & Elements & Occurrences & \multicolumn{1}{c}{ Values } & Depth \\
\hline \hline DBLP books & real & 1407 & 0.59 & 1.27 & 23 & 11456 & 6846 & 4 \\
\hline DBLP conf & real & 150885 & 58.93 & 125.83 & 29 & 1321593 & 567197 & 4 \\
\hline DBLP journals & real & 99475 & 37.19 & 86.76 & 23 & 973602 & 374196 & 5 \\
\hline HAM-RADIO & real & 882 & 360.96 & 730.93 & 23 & 14118079 & 2913795 & 4 \\
\hline Shakespeare & real & 37 & 7.53 & 21.51 & 28 & 179696 & 112766 & 6 \\
\hline Mondial & real & 1 & 2.04 & 5.46 & 49 & 85220 & 36929 & 6 \\
\hline Xmark $(100 \mathrm{Mb})$ & synth. & 1 & 113.79 & 324.98 & 77 & 2048193 & 416710 & 12 \\
\hline Xmark $(1 \mathrm{~Gb})$ & synth. & 1 & 1164.79 & 2052.00 & 77 & 20532805 & 2043572 & 12 \\
\hline \hline
\end{tabular}

Table 2. Datasets characteristics.

A brief description of the data sets content is the following:

- DBLP contains computer science bibliography information [12]. It consists of many small files, each representing a single record about publication (conference or journal paper, book).

- Shakespeare dataset is the XML version of the plays by Shakespeare [13].

- HAM-RADIO is the FCC Ham Radio database of the US Government's Federal Communications Commission [14].

- Mondial is freely available geographic database [15].

- Xmark datasets are synthetic and were generated by xmlgen generator [16]. The data models an auction website with large number of element and attribute types and high nesting of elements. We have selected standard $(100 \mathrm{Mb})$ and large $(1 \mathrm{~Gb})$ documents.

\subsection{Queries and Performance Metrics}

In order to study the tradeoffs of the join algorithm we carried out a series of comparative experiments. Query processing time was used as a major performance metric. The experiments evaluate the effect of several parameters on the performance of query processing: the size of the source XML files, number of elements and values, depth of the parsed tree, as well as the size of the query answer, number of elements

\begin{tabular}{|c|c|c|c|c|c|c|}
\hline \multirow{2}{*}{ \# } & \multirow{2}{*}{ XQuery expression } & \multirow{2}{*}{ Dataset } & \multicolumn{3}{|c|}{ Records } & \multirow{2}{*}{$\begin{array}{l}\mathrm{RPE} \\
\text { len }\end{array}$} \\
\hline & & & Input & Output & $\%$ & \\
\hline Q1 & /book/isbn & DBLP books & 705 & 621 & $88 \%$ & 2 \\
\hline Q2 & /inproceedings/cite & DBLP conf & 214837 & 102519 & $48 \%$ & 2 \\
\hline Q3 & /article/author & DBLP journals & 245748 & 245216 & $99 \%$ & 2 \\
\hline Q4 & /FMDataBase/FCCAmRadio/Address/City & HAM-RADIO & 2115906 & 2115906 & $100 \%$ & 3 \\
\hline Q5 & /PLAY/ACT/SPEECH/LINE & Shakespeare & 139083 & 138620 & $99 \%$ & 4 \\
\hline Q6 & /country/province/city/name & Mondial & 26032 & 6616 & $25 \%$ & 4 \\
\hline Q7 & \multirow{2}{*}{ /person/profile/interest/category } & Xmark (.1Gb) & 309588 & 97176 & $31 \%$ & 4 \\
\hline $\mathrm{Q} 8$ & & Xmark $(1 \mathrm{~Gb})$ & 1723670 & 977538 & $57 \%$ & 4 \\
\hline
\end{tabular}

Table 3. Description and parameters of the test queries. 
selected. The queries and their characteristics are given in Table 3 .

We chose several test queries based on the length of the regular path expression and number of matches. The column Input Records indicates the total number of processed records. The Output and "\%" columns show the number of records that match the query expression and their relative number against the processed records respectively. The RPE length represents the length of the query expression path. If all single element subexpressions of the query expression are different, then this number coincides with the number of select operations for the query, i.e. the number of input lists.

\subsection{Experimental Results}

The elapsed query time for the test datasets are shown in Figure 11. The cases with the query path length of more than 2 (queries Q4Q8) demonstrate improved performance of the sequence algorithm against the pair-wise algorithm.

The sequence join performs less effectively than the pair-wise join for the queries with the path length of 2 (queries Q1-Q3). This fact is in accordance with our time cost estimation shown in Figure 9.

For queries Q5-Q7, results are presented for different ways to break an n-way join into multiple binary joins. Sequential join algorithm has shown good performance against each of them. All the intermediate results for binary joins are materialised.

\section{Conclusion}

The structural join operation is intensely exploited for finding structural patterns within an XML database. Thus, an effective implementation of the structural join operation is essential for effective XML query processing.

We have developed the sequence join algorithm for regular path expressions. In contrast to pairwise approach, the algorithm takes several lists of elements as an input. It exploits the position of the element within XML document to compute structural relationships between elements fast. The algorithm checks structural pattern matching through all the input lists at once. Due to this, it does not generate nonexistent sub-results and hence eliminates creation of excessive intermediate data. Experimental evaluation of the algorithm shows that sequence join performs better than multiple binary joins for the queries with the expression length of 3 or more. It is true for the synthetic and real-world data queries of both small and large cardinality.

As a direction for future activities, it is worth to consider sequence joins in context of graphbased queries. This area poses many interesting
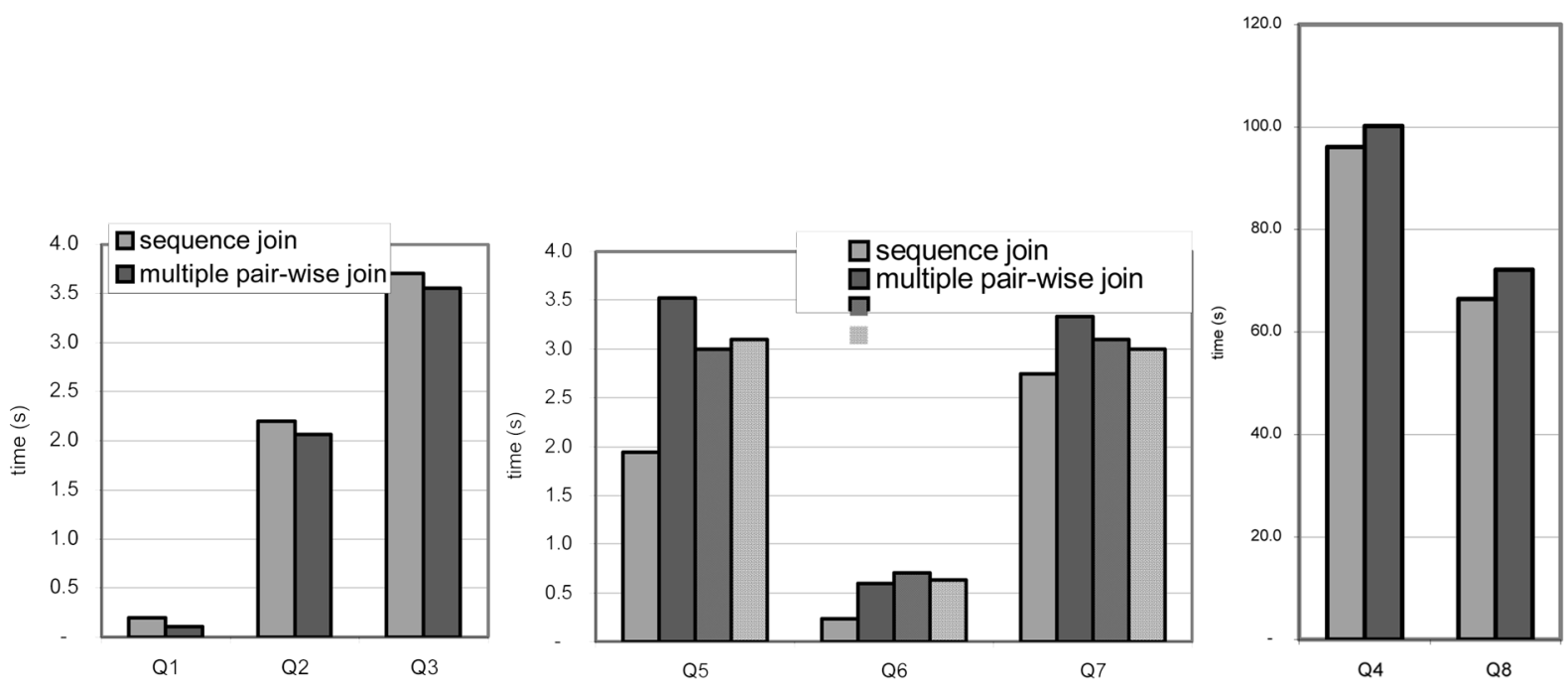

Fig. 11. Query performance comparison. 
problems, like handling graph-cycles (that are usually represented in the tree model as values of the link nodes). Another issue is processing the whole variety of XPath axes rather than ancestor-descendant or parent-child only.

\section{Acknowledgements}

This project is partly supported by ORS award. The authors would like to thank Jonathan Smith for his helpful comments on this paper.

\section{References}

[1] D. SucIU, On Database Theory and XML, in SIGMOD record, v.30, n.3, September 2001.

[2] S. Al-Khalifa, H.V. Jagadish, N. Koudas, J.M. PATEL, D. SRIVASTAVA, AND Y. WU, Structural Joins: A Primitive for Efficient XML Query Pattern Matching, Proceedings of the IEEE International Conference on Database Engineering (ICDE), 2002.

[3] Q. Li, B. MOON, INDEXING AND QUERYING XML DATA FOR REGULAR PATH EXPRESSIONS, Proceedings of the 27th VLDB Conference, Roma, Italy, 2001.

[4] L. KRISHNA, J. HARITSA, SphinX: Schemaconscious XML Indexing, Technical report TR2001-04, DSL/SERC.

[5] S. Abiteboul, D. Quass, J. McHugh, J. Widom, AND J. WIENER, The Lorel query language for semistructured data, International Journal on Digital Libraries, 1(1), pp. 68-88, April 1997.

[6] C. Zhang, J. F. Naughton, D. J. DeWitt, Q. LuO, G. M. LOHMAN, On Supporting Containment Queries in Relational Database Management Systems, Proceedings of the ACM SIGMOD Conference on Management Data, 2001.

[7] A. Deutsch, M. FernandeZ, D. Florescu, A. LEVY, D. SuCIU, A query language for XML, Computer Networks, 31(11-16), pp. 1155-1169, Amsterdam, Netherlands, 1999.

[8] T. Bray, J. PaOli, C.M. SPerberg-McQueEN, E. MALER, Extensible Markup Language (XML) 1.0 (Second Edition). W3C Recommendation, Technical report REC-XML-20001006. Available from http: //www . w3 . org/TR/REC-xml, October 2000 .

[9] J. Clark, S. DeRose, XML Path Language (XPath) 1.0. W3C Recommendation, Technical report REC-xpath-19991116. Available from http: //www.w3.org/TR/xpath, November 1999.
[10] N.Bruno, N.Koudas, D.Srivastava, Holistic Twig Joins: Optimal XML Pattern Matching, Proceedings of the ACM SIGMOD'02, 2002.

[11] S. BoAG, D. Chamberlin, M.F. FernandeZ, D. Florescu, J. RobIE, J. SimÉON, M. SteFANESCU, XQuery 1.0: An XML Query Language. W3C working draft, Technical report WDxquery-20020430, April 2002. Available from http: //www. w3.org/TR/xquery/.

[12] DBLP. Computer Science Bibliography, Available from http://www.informatik.uni-trier.de / ley/db/

[13] J. BosaK, The Plays of Shakespeare in XML. Available from http://xml.coverpages.org /bosakShakespeare200.html.

[14] HAM-RADIO. Available from ftp://ftp. ictcompress.com/pub/xmltestfiles/.

[15] W. MAY, Information Extraction and Integration with Florid: The Mondial Case Study, Technical report 131, Universität Freiburg, Institut für Informatik, 1999. Available from http://www . informatik. uni-freiburg.de/ may/ Mondial/.

[16] A. Schmidt, F. WAAS, M. Kersten, D. FloRESCU, I. MANOLESCU, M. J. CAREY, R. BuSSE, The XML benchmark project, Technical Report INS-R0103, CWI, April 2001. Available from http://monetdb.cwi.nl/xml/Benchmark/ benchmark.html.

[17] The Apache XML project. Xerces-C ++ is a validating XML parser. Available from http: //xml . apache.org/xerces-c/.

[18] Berkley BD. Available from http://www. sleepycat .com/.

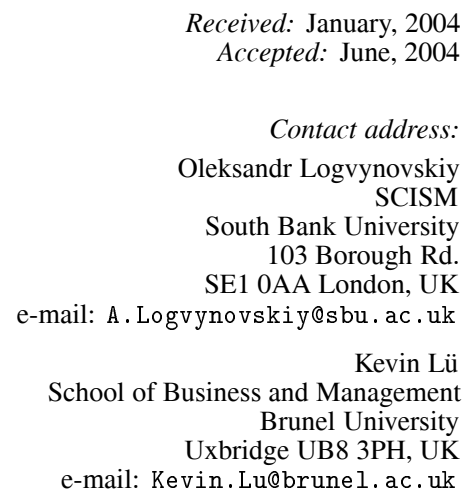

ALEXANDER A. LOGVYNOVSKIY MSc in Computing and BSc in Computing. Currently, he is a PhD research student at London South Bank University, UK. His project is about semi-structured data management and mining.

KeVIN J. LÜ PhD in Computer Science and BSc in Computer Science. $\mathrm{He}$ is a lecturer at Brunel University, UK. His current research areas of interests are data management, multi-agent system, intelligent data processing and enterprise information systems. 